\title{
Extended Stoney's Formula for a Film-Substrate Bilayer With the Effect of Interfacial Slip
}

\author{
Yin Zhang \\ State Key Laboratory of Nonlinear Mechanics \\ (LNM), \\ Institute of Mechanics, \\ Chinese Academy of Sciences, \\ Beijing 100080, P.R.C.
}

The curvature-stress relation is studied for a film-substrate bilayer with the effect of interfacial slip and compared with that of an ideal interface without interfacial slip. The interfacial slip together with the dimensions, elastic and interfacial properties of the film and substrate layers can cause a significant deviation of curvature-stress relation from that with an ideal interface. The interfacial slip also results in the so-called free edge effect that the stress, constraint force, and curvature vary dramatically around the free edges. The constant curvature as predicted by Stoney's formula and the Timoshenko model of an ideal interface is no longer valid for a bilayer with a nonideal interface. The models with the assumption of an ideal interface can also lead to an erroneous evaluation on the true stress state inside a bilayer with a nonideal interface. The extended Stoney's formula incorporating the effects of both the layer dimensions and interfacial slip is presented. [DOI: 10.1115/1.2745387]

\section{Introduction}

Almost all solid-state electronic components have the basic structure of a substrate as a platform for supporting various thin film structures [1]. Stress is of a great concern for the reliability of those composite structures $[1,2]$. Because the material properties of film and substrate such as Young's modulus, lattice parameters, coefficients of thermal expansion can be different and residual stress can build up during fabrication and processing, the resultant stresses inside the film and substrate can be different and result in the deflection of the composite structure to relax stress [3]. The following Stoney's 1909 formula [4] serves the cornerstone of relating the surface stress inside the film to the curvature of a composite structure

$$
\kappa_{\mathrm{St}}=\frac{6 f}{E_{2} t_{2}^{2}}
$$

$\kappa_{\mathrm{St}}$ is the curvature and $f$ is the force per unit length inside the film (when the film is very thin, $f$ is the surface stress [5]). $t_{2}$ is the substrate thickness and $E_{2}$ is the substrate effective Young's modulus. The applicability of the above Stoney's formula relies on several assumptions, which are well summarized by Freund et al. [6] as the following six: (1) both the film and substrate thicknesses are small compared to the lateral dimensions; (2) the film thickness is much less than the substrate thickness; (3) the substrate material is homogeneous, isotropic, and linearly elastic, and the film material is isotropic; (4) edge effect near the periphery of the substrate are inconsequential and all physical quantities are invariant under change in position parallel to the interface; (5) all stress components in the thickness direction vanish throughout the material; (6) the strains and rotations are infinitesimally small. Many models are developed to relax one or some of the above assumptions to extend Stoney's formula to a more generalized and realistic application, for example, by considering the effects of thin substrate [6-12], large deformation [6], nonisotropic stress [13], temperature gradient [14], stress gradient [7,15], residual axial force, boundary conditions, length $[5,16]$, diffusion effect [17], and plastic deformation [18]. However, all the analyses above [5-18] assume an ideal interface, i.e., no interfacial slip. In

Contributed by the Applied Mechanics Division of ASME for publication in the Journal of Applied Mechanics. Manuscript received September 6, 2006; final manuscript received March 19, 2007; published online January 11, 2008. Review conducted by Zhigang Suo. those models, the condition of no interfacial slip is enforced either explicitly by imposing the compatibility/continuity condition at the interface $[8,11,14]$ or implicitly by assuming one single strain/ displacement variable for both film and substrate layers $[5-7,9,10,12,13,15-18]$. Compared with an ideal interface (also called perfectly bonded interface [19] or coherent interface [20]), a nonideal interface results in the interfacial shear and normal stresses, which are generally zero in an ideal interface. Therefore, the overall deflection/curvature of the composite and the stress distribution inside it can by significantly affected. An interfacial shear stress due to temperature gradient is shown to exist in an ideal interface of a film-substrate bilayer by Huang and Rosakis [14], and this interfacial shear stress vanishes when there is no temperature gradient. However, when there is no temperature gradient, the interfacial stresses (both normal and shear) of a nonideal interface still exist and have an influence on the stress distribution inside the layers [3,21-24].

Because the strains inside two solid phases separated by an interface can be independent [20], the continuity condition of strain/displacement across an interface is a strong one, which allows no interfacial slip. During the fabrication and processing of film-substrate layered structures, such as chemical vapor deposition, wafer bonding, sputtering, doping/diffusion, implantation, thermal annealing process, heteroepitaxial film growth, etc., defects like dislocation [25,26], twin [27], cavities [25,28] appear. Therefore, the interface may not be composed of $100 \%$ well-fused bonds [28-30]. The formation of amorphous layer and dangling bonds in some regions between the two phases [29-31] also result in the weakly bonded interface areas. All these above will reduce the overall interface adhesion for sure [30]. The stress distribution inside the film calculated from the ideal interface model has been shown to deviate significantly from the experimental observation of a $\mathrm{Cu}-\mathrm{Si}$ composite with the size of several microns [23,24], which will also lead to a different deflection/curvature from that predicted by the ideal interface model.

The models of allowing interfacial slip are developed by Chen and Nelson [3] and Suhir [21,22], which are referred to as the shear-lag (S-L) model and lap-shear (L-S) model [24], respectively. Suhir's 1986 model gives a simple second order differential equation for the interfacial shear stress [21]. However, its drawback is noticed that the interfacial peeling stress (normal to the interface) cannot be self-equilibrated [32]. With the introduction of "compliant interface," Suhir's 1989 revised model [22] leads to a sixth order differential equation similar to that of Chen and 
Nelson [3]. The S-L model of Chen and Nelson is originally developed to study the stress distribution of two bonded joints. It assumes that there is an isotropic layer of finite thickness between the two joints, which plays the role of stress transfer between the two layers. This stress-transfer layer is extended to the concept of the interface layer $[23,24]$ as observed by high resolution transmission electron microscopy (HRTEM) that the 1-2 nm thick amorphous layer present at the interface of two crystal material layers $[23,24,28]$. The S-L model is shown to be equivalent to the damaged interface (DI) model [33] by Tullini [34]. Müller and Saúl give an in-depth discussion on various mechanisms causing the damaged/nonideal interface [35]. The effect, called the boundary layer effect or free edge effect unique to composite and not observed in homogeneous solids [19] arises, which is that the interfacial shear stress can be large and vary dramatically around the free edges (the constraint force and deflection/curvature which are related to the interfacial shear stress also share the similar characteristics). The interfacial stress is responsible for the failure (delamination, cracking) of composite structures [1,2,19], while, the ideal interface model as shown later in this paper cannot predict such boundary layer effect. The boundary layer effect is incorporated in the functions of interfacial stresses and their detailed solution procedures are given in this paper. This paper focuses on the effect of the interfacial slip on the overall deflection/curvature of the bilayer composite. The relation between the curvature and interfacial stresses is established by relaxing the aforementioned second, fourth and fifth assumptions given by Freund et al. [6]. The first, third, and sixth assumptions are retained, therefore, a beam model and related linear stress-strain relations can be applied. For the bilayer with a nonideal interface, the following two major results are presented in this paper: (1) the curvatures of the film and substrate with a nonideal interface can significantly deviate from the curvature predicated by the model of the ideal interface; (2) unlike that the film and substrate with an ideal interface share a common constant curvature; the curvatures of the film and substrate with a nonideal interface are different in general and vary with the length. These two results have a significant impact on the interpretation of the experimental data of the curvature-based measurement.

\section{Model Development}

2.1 Strain Distribution due to Lattice Mismatch. The lattice mismatch induced deflection of a film-substrate composite fabricated by heteroepitaxial growth is analyzed as an example to demonstrate the effect of interfacial slip on curvature. The strains inside the film and substrate layers are first calculated by assuming no composite deflection. The composite deflection with the presence of this calculated strain distribution is then derived in the following two models with and without interfacial slip. The derivation approach of composite deflection presented here is general, which can be applied to the analysis of composite deflection induced by other mechanisms. The lattice mismatch induced strains inside the film and substrate have the following relations [17]:

$$
\begin{gathered}
\epsilon_{f}-\epsilon_{s}=\epsilon_{m} \\
E_{1} t_{1} \epsilon_{f}+E_{2} t_{2} \epsilon_{s}=0
\end{gathered}
$$

$\epsilon_{f}$ and $\epsilon_{s}$ are the strains induced by lattice mismatch in the film and substrate, respectively. $\epsilon_{m}$ is the mismatch strain defined as $\epsilon_{m}=\left(a_{s}-a_{f}\right) / a_{f}[7]\left(a_{f}\right.$ and $a_{s}$ are the lattice parameters of the film and substrate, respectively). $E_{1}$ and $E_{2}$ are the effective Young's moduli of the film and substrate. When the composite is a plate structure and under biaxial stress loading, $E_{1}=Y_{1} /\left(1-\nu_{1}\right)$ and $E_{2}=Y_{2} /\left(1-\nu_{2}\right)$ are the biaxial moduli [7] $\left(Y_{1}\right.$ and $Y_{2}$ are the Young's moduli of the film and substrate, respectively. $\nu_{1}$ and $\nu_{2}$ are their Poisson's ratios). When the composite plate bends to a cylindrical surface, $E_{1}=Y_{1} /\left(1-\nu_{1}^{2}\right)$ and $E_{2}=Y_{2} /\left(1-\nu_{2}^{2}\right)$ [36]. When the composite is a beam structure, $E_{1}=Y_{1}$ and $E_{2}=Y_{2} \cdot t_{1}$ (a) Two coordinate sets at the mid-planes of two layers

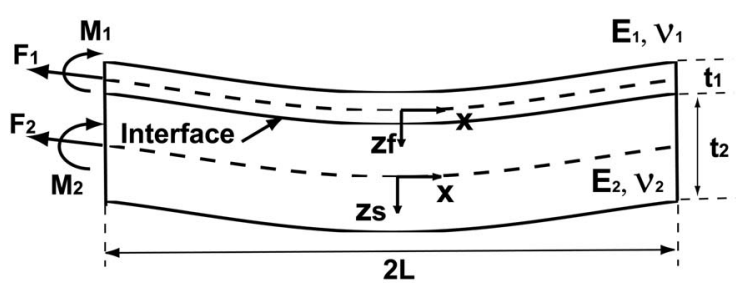

\section{(b) Illustration of interfacial normal and shear stresses of S-L model}

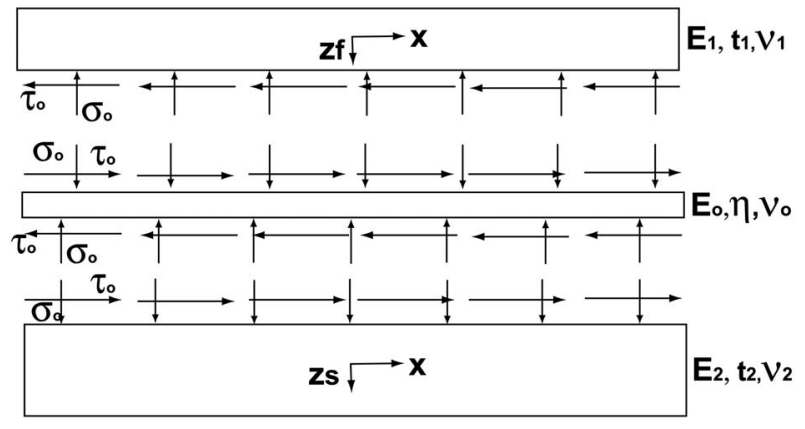

Fig. 1 (a) Two coordinate systems at the mid-planes of two layers. (b) Illustration of interfacial normal stress $\left(\sigma_{o}\right)$ and shear stress $\left(\tau_{o}\right)$ in the S-L model.

and $t_{2}$ as shown in Fig. 1(a) are the thickness of the film and substrate. The first equation in Eq. (2) is the compatibility condition and the second one is the Newton's third law. From Eq. (2), $\epsilon_{f}$ and $\epsilon_{s}$ are solved as follows:

$$
\begin{gathered}
\epsilon_{f}=\epsilon_{m} \frac{E_{2} t_{2}}{E_{1} t_{1}+E_{2} t_{2}} \\
\epsilon_{s}=-\epsilon_{m} \frac{E_{1} t_{1}}{E_{1} t_{1}+E_{2} t_{2}}
\end{gathered}
$$

Clearly, $\epsilon_{f}$ and $\epsilon_{s}$ have the opposite signs, which implies that there is a bending moment inside the film-substrate bilayer and the bilayer must deflect. The following derivation is about how the bilayer accommodates this bending moment by deflecting with and without interfacial slip.

2.2 Timoshenko Model. The Timoshenko model [8] is essentially a beam model. There is other method of solving this composite bending problem, such as elasticity approach [19]. Because the Timoshenko model uses the curvature as the unknown variable, it has the advantage that the curvature is directly solved.

The equilibrium requires the balance of both force and moment, which gives the following two equations:

$$
F_{1}+F_{2}=0
$$

and

$$
M_{1}+M_{2}-F_{1} \frac{t_{1}}{2}+F_{2} \frac{t_{2}}{2}=0
$$

$F_{1}$ and $M_{1}$ are the force and moment per unit width acting inside the film layer as shown in Fig. $1(a) . F_{2}$ and $M_{2}$ are those inside the substrate layer. From Eq. (4), the following equation is derived:

$$
F_{1}=P(x)=-F_{2}
$$

Substitute Eq. (6) into Eq. (5), it gives 


$$
\frac{P(x)\left(t_{1}+t_{2}\right)}{2}=M_{1}+M_{2}
$$

The longitudinal normal strains of the two layers are expressed as the following:

$$
\begin{gathered}
\frac{d u_{1}(x, z)}{d x}=\epsilon_{f}+\frac{P(x)}{E_{1} t_{1}}+\frac{z}{\rho} \\
-\frac{t_{1}}{2} \leqslant z \leqslant \frac{t_{1}}{2} \\
\frac{d u_{2}(x, z)}{d x}=\epsilon_{s}-\frac{P(x)}{E_{2} t_{2}}+\frac{z}{\rho} \\
-\frac{t_{2}}{2} \leqslant z \leqslant \frac{t_{2}}{2}
\end{gathered}
$$

$u_{1}(x, z)$ and $u_{2}(x, z)$ are the longitudinal displacements of the film and substrate, respectively. As shown in Fig. 1(a), two sets of coordinates are used in the Timoshenko model. $z_{f}$ and $z_{s}$ start from the midplanes of the film and substrate, respectively. $P(x)$ is the constraint force per unit width. $\rho$ is the radius of curvature. Because curvature $\kappa=1 / \rho$, Eq. (8) assumes that the film and substrate share a common curvature [32].

The bending moment $\left(M_{i}, i=1,2\right)$ has the following relation with $\rho$ :

$$
M_{i}=\frac{E_{i} t_{i}^{3}}{12 \rho}
$$

Substitute Eq. (9) into Eq. (7), the following equation is obtained:

$$
\frac{1}{\rho}=\frac{6 P(x)\left(t_{1}+t_{2}\right)}{E_{1} t_{1}^{3}+E_{2} t_{2}^{3}}
$$

The compatibility condition at the interface requires that

$$
\epsilon_{f}+\frac{P(x)}{E_{1} t_{1}}+\frac{t_{1}}{2 \rho}=\epsilon_{s}-\frac{P(x)}{E_{2} t_{2}}-\frac{t_{2}}{2 \rho}
$$

Equation (11) physically indicates the continuity of strain at the interface, i.e., that there is no slip between the two layers. From Eq. (11), $P(x)$ is solved as a constant as follows:

$$
P(x)=\frac{-\epsilon_{m}}{1 / E_{1} t_{1}+1 / E_{2} t_{2}+3\left(t_{1}+t_{2}\right)^{2} /\left(E_{1} t_{1}^{3}+E_{2} t_{2}^{3}\right)}
$$

Substitute this $P(x)$ of Eq. (12) into Eq. (10) and the curvature $\left(\kappa_{T}\right)$ is solved also as a constant as the following:

$$
\kappa_{T}=\frac{1}{\rho}=\frac{\kappa_{\mathrm{St}}\left(1+\frac{t_{1}}{t_{2}}\right)}{1+4 \frac{t_{1} E_{1}}{t_{2} E_{2}}+6 \frac{t_{1}^{2} E_{t}}{t_{2}^{2} E_{2}}+4 \frac{t_{1}^{3} E_{1}}{t_{2}^{3} E_{2}}+\frac{t_{1}^{4} E_{1}^{2}}{t_{2}^{4} E_{2}^{2}}}
$$

$\kappa_{\mathrm{St}}$ is the Stoney's formula of Eq. (1) when $f$ is set as $f$ $=-\epsilon_{m} E_{1} t_{1}$. The Timoshenko model is a free body diagram analysis with the introduction of constraint force $P(x)$. The curvature of Eq. (13) is exactly the same as that derived by Freund and Suresh [17] who use an energy approach. In Freund and Suresh's derivation [7], there is no constraint force assumed, but one displacement variable is used for both film and substrate layer (therefore, the continuity of displacement and strain at the interface is automatically satisfied), which is equivalent to the enforcement of no interfacial slip in Eq. (11). It is also noticed that $\lim _{t_{1} / t_{2} \rightarrow 0} \kappa_{T}$ $=\kappa_{\mathrm{St}}$ and the Timoshenko model in essence extends the Stoney formula by relaxing the aforementioned second assumption given by Freund et al. [6].
2.3 S-L Model. As the S-L model is demonstrated to fit the experimental observation much better than the L-S model [23,24], the S-L model is adopted here to study the interfacial slip effect. The S-L model is rather complex and here an outline of its derivation is given for reader to better understand. In the S-L model, an interfacial layer is assumed. The interfacial normal stress $\left(\sigma_{o}(x)\right)$ and shear stress $\left(\tau_{o}(x)\right)$ due to interfacial slip is illustrated in Fig. 1(b). With interfacial slip, the constraint condition of Eq. (11) is invalid. $\sigma_{o}(x)$ and $\tau_{o}(x)$ are related to the longitudinal and transverse displacements of the film and substrate layers, respectively. The presence of $\sigma_{o}(x)$ and $\tau_{o}(x)$ changes the equation of equilibrium of each layer as reflected in the following derivations. Because the longitudinal and transverse displacements of the film and substrate layers are not independent of each other, $\sigma_{o}(x)$ and $\tau_{o}(x)$ are not two independent variables, either. Therefore, the major effort in the following derivation is actually to decouple $\sigma_{o}(x)$ and $\tau_{o}(x)$ for the solution purpose, which also results in the two sixth order differential governing equations for $\sigma_{o}(x)$ and $\tau_{o}(x)$.

$\sigma_{o}(x)$ and $\tau_{o}(x)$ have the following expressions:

$$
\begin{gathered}
\sigma_{o}(x)=\frac{E_{o}}{\eta}\left[v_{2}(x)-v_{1}(x)\right] \\
\tau_{o}(x)=\frac{G_{o}}{\eta}\left[u_{1}\left(x, \frac{t_{1}}{2}\right)-u_{2}\left(x,-\frac{t_{2}}{2}\right)\right]
\end{gathered}
$$

$E_{o}, G_{o}$ are the Young's modulus and shear modulus of the interface layer, respectively. $\eta$ is the thickness of the interface layer. In the S-L model, $E_{o}, G_{o}$, and $\eta$ are fitting factors [23,24]. The actual varying parameters in the S-L model are $E_{o} / \eta$ and $G_{o} / \eta$. $u_{1}\left(x, t_{1} / 2\right)$ and $u_{2}\left(x,-t_{2} / 2\right)$ are the longitudinal displacements of the film layer and substrate layer at the interface, respectively. $v_{1}(x)$ and $v_{2}(x)$ are the transverse displacements of the midplanes of the layers. The transverse displacement $\left(v_{i}(x)\right)$ in the S-L model is independent of $z$. As indicated in Fig. 1(b) $\sigma_{o}(x)$ is perpendicular to the interface and the fifth assumption of the Stoney formula [6] is clearly violated. Also as shown later, the boundary conditions are used to find $\sigma_{o}(x)$ and $\tau_{o}(x)$ and the edge effects are thus incorporated into their solutions; $\sigma_{o}(x)$ and $\tau_{o}(x)$ are the functions of $x$ and they vary along the direction parallel to the interface. The fourth assumption is also violated. The following derivation as the above Timoshenko model does not need the second assumption of very thin film thickness, either. As mentioned above, the interfacial normal and shear stresses do not exist in an ideal interface case. In Eq. (8) $P$ is a normal constant constraint force parallel to the interface. In the model of a film-viscous layer-rigid substrate by Huang and Suo [37,38], the pressure that drives the flow of viscous layer also acts on the film in the direction perpendicular to the interface and the flow itself provides the shear stress (parallel to the interface) to the film layer, which resembles the functions of $\sigma_{o}(x)$ and $\tau_{o}(x)$. Although Huang and Suo assume that the no-slip boundary condition (at the viscous layer-rigid substrate interface) and the elastic film is bonded to the viscous layer $[37,38]$, the flow of the viscous layer in fact causes the slip between the film and substrate.

In the S-L model, the equilibrium of moment is

$$
\begin{aligned}
& \frac{d M_{1}(x)}{d x}-V_{1}(x)-\frac{t_{1}}{2} \tau_{o}(x)=0 \\
& \frac{d M_{2}(x)}{d x}-V_{2}(x)-\frac{t_{2}}{2} \tau_{o}(x)=0
\end{aligned}
$$

$M_{1}(x)$ and $M_{2}(x)$ are the moments acting in layer 1 and 2, respectively. $V_{i}(i=1,2)$ stands for the vertical shear force per unit width. The vertical force equilibrium requires 


$$
\begin{aligned}
& d V_{1}(x)+\sigma_{o}(x) d x=0 \\
& d V_{2}(x)-\sigma_{o}(x) d x=0
\end{aligned}
$$

Differentiating Eq. (16) and using Eq. (17), the following is derived:

$$
\begin{aligned}
& \frac{d^{2} M_{1}(x)}{d x^{2}}+\sigma_{o}(x) d x-\frac{t_{1}}{2} \frac{d \tau_{o}(x)}{d x}=0 \\
& \frac{d^{2} M_{2}(x)}{d x^{2}}-\sigma_{o}(x) d x-\frac{t_{2}}{2} \frac{d \tau_{o}(x)}{d x}=0
\end{aligned}
$$

The moment and curvature radius have the following relation:

$$
M_{i}(x)=\frac{D_{i}}{\rho_{i}(x)}=-D_{i} \frac{d^{2} v_{i}(x)}{d x^{2}}
$$

Here $\rho_{i} \quad(i=1,2)$ is the radius of curvature and $1 / \rho_{i}(x)$ $=-d^{2} v_{i}(x) / d x^{2}$, the minus sign is due to the coordinate system as shown in Fig. 1(a) $[8,24,36]$. It should be kept in mind that in general $d^{2} v_{1}(x) / d x^{2} \neq d^{2} v_{2}(x) / d x^{2}$ for the composite with interfacial slip. $D_{i}(i=1,2)$ is the bending stiffness per unit width of layer $i$ defined as $D_{i}=E_{i} t_{i}^{3} / 12$. Substituting Eq. (19) into Eq. (18), the following is derived:

$$
\begin{aligned}
& -D_{1} \frac{d^{4} v_{1}(x)}{d x^{4}}+\sigma_{o}(x)-\frac{t_{1}}{2} \frac{d \tau_{o}(x)}{d x}=0 \\
& -D_{2} \frac{d^{4} v_{2}(x)}{d x^{4}}-\sigma_{o}(x)-\frac{t_{2}}{2} \frac{d \tau_{o}(x)}{d x}=0
\end{aligned}
$$

Differentiating Eq. (14) four times and using the expressions of $d^{4} v_{1} / d x^{4}$ and $d^{4} v_{2} / d x^{4}$ derived from Eq. (20), the following is obtained:

$$
\frac{d^{4} \sigma_{o}(x)}{d x^{4}}+\frac{E_{o} b}{\eta} \sigma_{o}(x)=\frac{E_{o} a}{\eta} \frac{d \tau_{o}(x)}{d x}
$$

$a$ and $b$ are defined as follows:

$$
\begin{gathered}
a=\frac{1}{2}\left(\frac{t_{1}}{D_{1}}-\frac{t_{2}}{D_{2}}\right) \\
b=\frac{1}{D_{1}}+\frac{1}{D_{2}}
\end{gathered}
$$

The longitudinal strains of the two layers in the S-L model have the following expressions:

$$
\begin{aligned}
& \frac{d u_{1}(x, z)}{d x}=\epsilon_{f}+\frac{P(x)}{E_{1} t_{1}}-z \frac{d^{2} v_{1}(x)}{d x^{2}}-\frac{t_{1}}{2} \leqslant z \leqslant \frac{t_{1}}{2} \\
& \frac{d u_{2}(x, z)}{d x}=\epsilon_{s}-\frac{P(x)}{E_{2} t_{2}}-z \frac{d^{2} v_{2}(x)}{d x^{2}}-\frac{t_{2}}{2} \leqslant z \leqslant \frac{t_{2}}{2}
\end{aligned}
$$

$P(x)$ is also an unknown constraint axial force. At the interface, the longitudinal strains are as follows:

$$
\begin{aligned}
& \frac{d u_{1}\left(x, \frac{t_{1}}{2}\right)}{d x}=\epsilon_{f}+\frac{P(x)}{E_{1} t_{1}}-\frac{t_{1}}{2} \frac{d^{2} v_{1}(x)}{d x^{2}} \\
& \frac{d u_{2}\left(x,-\frac{t_{2}}{2}\right)}{d x}=\epsilon_{s}-\frac{P(x)}{E_{2} t_{2}}+\frac{t_{2}}{2} \frac{d^{2} v_{2}(x)}{d x^{2}}
\end{aligned}
$$

Differentiating Eq. (15), gives

$$
\frac{d \tau_{o}(x)}{d x}=\frac{G_{o}}{\eta}\left[\frac{d u_{1}\left(x, \frac{t_{1}}{2}\right)}{d x}-\frac{d u_{2}\left(x,-\frac{t_{2}}{2}\right)}{d x}\right]
$$

Substituting Eq. (24) into Eq. (25), gives

$$
\frac{d \tau_{o}(x)}{d x}=\frac{G_{o}}{\eta}\left[\left(\frac{t_{1}^{2}}{12 D_{1}}+\frac{t_{2}^{2}}{12 D_{2}}\right) P(x)-\frac{t_{1}}{2} \frac{d^{2} v_{1}(x)}{d x^{2}}-\frac{t_{2}}{2} \frac{d^{2} v_{2}(x)}{d x^{2}}-\Delta\right]
$$

Here $\Delta=\epsilon_{s}-\epsilon_{f}$. Differentiating Eq. (26) twice and using both Eq. (20) and the fact of $d P(x) / d x=\tau_{o}(x)$, gives

$$
\frac{d^{3} \tau_{o}(x)}{d x^{3}}-\frac{G_{o} c}{\eta} \frac{d \tau_{o}(x)}{d x}=-\frac{G_{o} a}{\eta} \sigma_{o}(x)
$$

and $c=\left(t_{1}^{2} / D_{1}+t_{2}^{2} / D_{2}\right) / 3$. Differentiating Eq. (21) twice and using Eqs. (21) and (27) again, leads to

$$
\frac{d^{6} \sigma_{o}(x)}{d x^{6}}-\frac{G_{o} c}{\eta} \frac{d^{4} \sigma_{o}(x)}{d x^{4}}+\frac{E_{o} b}{\eta} \frac{d^{2} \sigma_{o}(x)}{d x^{2}}-\frac{E_{o} G_{o}\left(b c-a^{2}\right)}{\eta^{2}} \sigma_{o}(x)=0
$$

Equation (28) is the uncoupled governing equation of interfacial normal stress $\sigma_{o}(x)$. To derive the uncoupled governing equation of interfacial shear stress $\tau_{o}(x)$, Eq. (27) is differentiated four times and Eqs. (21) and (27) are used again. The seventh order differential equation is obtained as follows:

$$
\frac{d^{7} \tau_{o}(x)}{d x^{7}}-\frac{G_{o} c}{\eta} \frac{d^{5} \tau_{o}(x)}{d x^{5}}+\frac{E_{o} b}{\eta} \frac{d^{3} \tau_{o}(x)}{d x^{3}}-\frac{G_{o} E_{o}\left(b c-a^{2}\right)}{\eta^{2}} \frac{d \tau_{o}(x)}{d x}=0
$$

Integrate Eq. (29) once with the use of $\tau_{o}(0)=0$ because the interfacial shear stress is an odd function $[3,24]$, the following sixth order differential equation is derived

$$
\frac{d^{6} \tau_{o}(x)}{d x^{6}}-\frac{G_{o} c}{\eta} \frac{d^{4} \tau_{o}(x)}{d x^{4}}+\frac{E_{o} b}{\eta} \frac{d^{2} \tau_{o}(x)}{d x^{2}}-\frac{E_{o} G_{o}\left(b c-a^{2}\right)}{\eta^{2}} \tau_{o}(x)=0
$$

It must be emphasized that the two uncoupled Eqs. (28) and (30) are not independent of each other and they are related to each other by Eq. (21).

2.4 Solutions of $\sigma_{o}(x), \tau_{o}(x)$, and Curvatures. $\sigma_{o}(x)$ of Eq. (28) has the following solution form [3,24]:

$$
\begin{aligned}
\sigma_{o}(x)= & A_{1} \cosh \left(\beta_{1} x\right)+A_{2} \sinh \left(\beta_{1} x\right)+A_{3} \cosh \left(\beta_{h} x\right) \cos \left(\beta_{v} x\right) \\
& +A_{4} \sinh \left(\beta_{h} x\right) \cos \left(\beta_{v} x\right)+A_{5} \sinh \left(\beta_{h} x\right) \sin \left(\beta_{v} x\right) \\
& +A_{6} \cosh \left(\beta_{h} x\right) \sin \left(\beta_{v} x\right)
\end{aligned}
$$

Here $A_{i} \mathrm{~s}(i=1-6)$ are the unknown constants to be determined by the boundary conditions. $\beta_{1}, \beta_{h}$, and $\beta_{v}$ are the eigenvalues solved from the characteristic equation of Eq. (28). The following parameters are defined to express the eigenvalues:

$$
\begin{gathered}
\Phi_{0}=\frac{c}{3} \frac{G_{o}}{\eta} \quad \Phi_{1}=\frac{b}{3} \frac{E_{o}}{\eta}-\frac{c^{2}}{9}\left(\frac{G_{o}}{\eta}\right)^{2} \\
\Phi_{2}=\frac{1}{3}\left[c^{3}\left(\frac{G_{o}}{\eta}\right)^{3}-\frac{27}{2} a^{2} \frac{E_{o}}{\eta} \frac{G_{o}}{\eta}+9 b c \frac{E_{o}}{\eta} \frac{G_{o}}{\eta}+\frac{R_{1}}{2 \eta^{6}}\right]^{1 / 3}
\end{gathered}
$$

$R_{1}$ is defined as follows:

$$
\begin{aligned}
R_{1}= & {\left[4\left(3 b E_{o} \eta^{3}-c^{2} G_{o}^{2} \eta^{2}\right)^{3}+\left(2 c^{3} G_{o}^{3} \eta^{3}-27 a^{2} E_{o} G_{o} \eta^{4}\right.\right.} \\
& \left.\left.+18 b c E_{o} G_{o} \eta^{4}\right)^{2}\right]^{1 / 2}
\end{aligned}
$$

$y_{1}, y_{h}$, and $y_{v}$ are defined as 
$y_{1}=\Phi_{0}-\frac{\Phi_{1}}{\Phi_{2}}+\Phi_{2} \quad y_{h}=\Phi_{0}+\frac{1}{2}\left(\frac{\Phi_{1}}{\Phi_{2}}-\Phi_{2}\right) \quad y_{v}=\frac{\sqrt{3}}{2}\left(\frac{\Phi_{1}}{\Phi_{2}}+\Phi_{2}\right)$

Now $\beta_{1}, \beta_{h}$, and $\beta_{v}$ are defined as

$$
\begin{gathered}
\beta_{1}=\sqrt{y_{1}} \quad \beta_{h}=\sqrt{\frac{\sqrt{y_{h}^{2}+y_{v}^{2}}}{1+\tan ^{2}\left[\frac{1}{2} \tan ^{-1}\left(y_{h} / y_{v}\right)\right]}} \\
\beta_{v}=\beta_{h} \tan \left[\frac{1}{2} \tan ^{-1}\left(y_{v} / y_{h}\right)\right]
\end{gathered}
$$

The symmetry condition requires $\sigma_{o}(x)$ to be an even function $[3,24]$, therefore Eq. (31) changes as follows:

$$
\begin{aligned}
\sigma_{o}(x)= & A_{1} \cosh \left(\beta_{1} x\right)+A_{3} \cosh \left(\beta_{h} x\right) \cos \left(\beta_{v} x\right) \\
& +A_{5} \sinh \left(\beta_{h} x\right) \sin \left(\beta_{v} x\right)
\end{aligned}
$$

The 3 boundary conditions for Eq. (36) are the following [3,24]:

$$
\begin{gathered}
\int_{-L}^{L} \sigma_{o}(x) d x=0 \quad \frac{d^{2} \sigma_{o}(L)}{d x^{2}}=0 \\
\frac{d^{4} \sigma_{o}(L)}{d x^{4}}-\frac{E_{o} b}{\eta} \sigma_{o}(L)=-\frac{E_{o} a}{\eta} \frac{G_{o} \Delta}{\eta}
\end{gathered}
$$

$-L \leqslant x \leqslant L$ and $2 L$ is the beam length. Here the boundary conditions are expressed by $\sigma_{o}$ and their physical meaning cannot easily be told. Physically, the first boundary condition above indicates that the vertical shear force is zero at the free edge. The second and third ones involve the fact that both the axial force and bending moment are zero at the free edges $[3,24]$. The 3 boundary conditions of Eq. (37) written in $A_{i}$ s are the following 3 equations:

$$
\begin{aligned}
& \frac{\sinh \left(\beta_{1} L\right)}{\beta_{1}} A_{1}+\left[\frac{\beta_{v}}{\beta_{h}^{2}+\beta_{v}^{2}} \cosh \left(\beta_{h} L\right) \sin \left(\beta_{v} L\right)\right. \\
& \left.+\frac{\beta_{h}}{\beta_{h}^{2}+\beta_{v}^{2}} \sinh \left(\beta_{h} L\right) \cos \left(\beta_{v} L\right)\right] A_{3} \\
& +\left[\frac{\beta_{h}}{\beta_{h}^{2}+\beta_{v}^{2}} \cosh \left(\beta_{h} L\right) \sin \left(\beta_{v} L\right)\right. \\
& \left.-\frac{\beta_{v}}{\beta_{h}^{2}+\beta_{v}^{2}} \sinh \left(\beta_{h} L\right) \cos \left(\beta_{v} L\right)\right] A_{5}=0 \\
& \beta_{1}^{2} \cosh \left(\beta_{1} L\right) A_{1}+\left[\left(\beta_{h}^{2}-\beta_{v}^{2}\right) \cosh \left(\beta_{h} L\right) \cos \left(\beta_{v} L\right)\right. \\
& \left.-2 \beta_{h} \beta_{v} \sinh \left(\beta_{h} L\right) \sin \left(\beta_{v} L\right)\right] A_{3} \\
& +\left[\left(\beta_{h}^{2}-\beta_{v}^{2}\right) \sinh \left(\beta_{h} L\right) \sin \left(\beta_{v} L\right)\right. \\
& \left.+2 \beta_{h} \beta_{v} \cosh \left(\beta_{h} L\right) \cos \left(\beta_{v} L\right)\right] A_{5}=0 \\
& {\left[\left(\beta_{1}^{4}+\frac{E_{o} b}{\eta}\right) \cosh \left(\beta_{1} L\right)\right] A_{1}+\left[\left(\beta_{h}^{2}-\beta_{v}^{2}\right)^{2}-4 \beta_{h}^{2} \beta_{v}^{2}\right.} \\
& \left.+\frac{E_{o} b}{\eta}\right) \cosh \left(\beta_{h} L\right) \cos \left(\beta_{v} L\right)-4 \beta_{h} \beta_{v}\left(\beta_{h}^{2}\right. \\
& \left.\left.-\beta_{v}^{2}\right) \sinh \left(\beta_{h} L\right) \sin \left(\beta_{v} L\right)\right] A_{3}+\left[\left(\beta_{h}^{2}-\beta_{v}^{2}\right)^{2}-4 \beta_{h}^{2} \beta_{v}^{2}\right. \\
& \left.+\frac{E_{o} b}{\eta}\right) \sinh \left(\beta_{h} L\right) \sin \left(\beta_{v} L\right)+4 \beta_{h} \beta_{v}\left(\beta_{h}^{2}\right. \\
& \left.\left.-\beta_{v}^{2}\right) \cosh \left(\beta_{h} L\right) \cos \left(\beta_{v} L\right)\right] A_{5}=-\frac{E_{o} a}{\eta} \frac{G_{o} \Delta}{\eta}
\end{aligned}
$$

The 3 boundary conditions above uniquely determine the values of $A_{1}, A_{2}$, and $A_{3}$.

For the solution of interfacial shear stress $\tau_{o}(x)$ of Eq. (30), the solution has the following form:

$$
\begin{aligned}
\tau_{o}(x)= & C_{1} \sinh \left(\beta_{1} x\right)+C_{2} \sinh \left(\beta_{h} x\right) \cos \left(\beta_{v} x\right) \\
& +C_{3} \cosh \left(\beta_{h} x\right) \sin \left(\beta_{v} x\right)+C_{4} \cosh \left(\beta_{h} x\right) \cos \left(\beta_{v} x\right) \\
& +C_{5} \sinh \left(\beta_{h} x\right) \sin \left(\beta_{v} x\right)+C_{6} \cosh \left(\beta_{1} x\right)
\end{aligned}
$$

Here $C_{i} \mathrm{~s}(i=1-6)$ are the unknown constants to be determined by the boundary conditions. While, in order to keep $\tau_{o}(x)$ as an odd function [3,24], Eq. (41) changes to the following form:

$$
\begin{aligned}
\tau_{o}(x)= & C_{1} \sinh \left(\beta_{1} x\right)+C_{2} \sinh \left(\beta_{h} x\right) \cos \left(\beta_{v} x\right) \\
& +C_{3} \cosh \left(\beta_{h} x\right) \sin \left(\beta_{v} x\right)
\end{aligned}
$$

$C_{i}(i=1-3)$ are correlated to $A_{i} \mathrm{~s}$ via Eq. (21). $C_{i} \mathrm{~s}$ are expressed in $A_{i} \mathrm{~s}$ as

$$
\begin{gathered}
C_{1}=\frac{1}{\beta_{1}} \frac{\eta}{E_{o} a}\left(\beta_{1}^{4}+\frac{E_{o} b}{\eta}\right) A_{1} \quad C_{2}=\frac{\eta}{E_{o} a}\left(\gamma_{1} A_{3}-\gamma_{2} A_{5}\right) \\
C_{3}=\frac{\eta}{E_{o} a}\left(\gamma_{1} A_{5}+\gamma_{2} A_{3}\right)
\end{gathered}
$$

with the definition of $\gamma_{1}$ and $\gamma_{2}$ as

$$
\begin{aligned}
& \gamma_{1}=\beta_{h}\left[\frac{E_{o} b}{\eta\left(\beta_{h}^{2}+\beta_{v}^{2}\right)}+\beta_{h}^{2}-3 \beta_{v}^{2}\right] \\
& \gamma_{2}=\beta_{v}\left[\frac{E_{o} b}{\eta\left(\beta_{h}^{2}+\beta_{v}^{2}\right)}+\beta_{v}^{2}-3 \beta_{h}^{2}\right]
\end{aligned}
$$

Once $\sigma_{o}(x)$ and $\tau_{o}(x)$ are solved, $\kappa_{f}$ can be obtained as follows by integrating the first equation of Eq. (16):

$$
\kappa_{f}=-\frac{d^{2} v_{1}(x)}{d x^{2}}=-\frac{1}{D_{1}} \int_{-L}^{x} \int_{-L}^{x} \sigma_{o}(x) d x d x+\frac{t_{1}}{2 D_{1}} \int_{-L}^{x} \tau_{o}(x) d x
$$

During the derivation of Eq. (45), the facts that $M_{1}$ $=-D_{1} d^{2} v_{1} / d x^{2}$ in Eq. (19) and $d V_{1} / d x=-\sigma_{o}(x)$ in Eq. (17) are also used. Similarly, the curvature of the substrate is found by integrating the second equation of Eq. (16),

$$
\kappa_{s}=-\frac{d^{2} v_{2}(x)}{d x^{2}}=\frac{1}{D_{2}} \int_{-L}^{x} \int_{-L}^{x} \sigma_{o}(x) d x d x+\frac{t_{2}}{2 D_{2}} \int_{-L}^{x} \tau_{o}(x) d x
$$

Generally, $\kappa_{f} \neq \kappa_{s}$ in the S-L model in contrast to $\kappa_{f}=\kappa_{s}=\kappa_{T}$ of the Timoshenko model. A more dramatic case is demonstrated in the model of a (soft) film-viscous layer-rigid substrate $[37,38]$ that the soft film is buckled with relatively large wave number and the rigid substrate remains flat. Also, the above curvatures of the film and substrate in the S-L model vary along the beam span in contrast to the constants of the Timoshenko model. Huang and Rosakis also summarize six assumptions for the applicability of the Stoney formula and their sixth is that "all surviving stress and curvature components are spatially constant over the plate system's surface, a situation which is often violated in practice" [14]. Clearly, here the interfacial slip is one of the mechanisms responsible for such violation. With the solution of $\tau_{o}(x)$ and $P(x)$ $=\int_{-L}^{x} \tau_{o} d x$ [24], the strain/stress inside the film and substrate can also be evaluated via Eq. (23).

\section{Results and Discussion}

Here the film is germanium with $Y_{1}=105.08 \mathrm{GPa}, \nu_{1}=0.26$, and $a_{f}=0.56574 \mathrm{~nm}$; the substrate is silicon with $Y_{2}=150 \mathrm{GPa}, \nu_{2}$ $=0.17$, and $a_{s}=0.54306 \mathrm{~nm}[7] . \epsilon_{m} \approx-4 \%$. The interface layer is 
(a) Definations of different radii of curvature

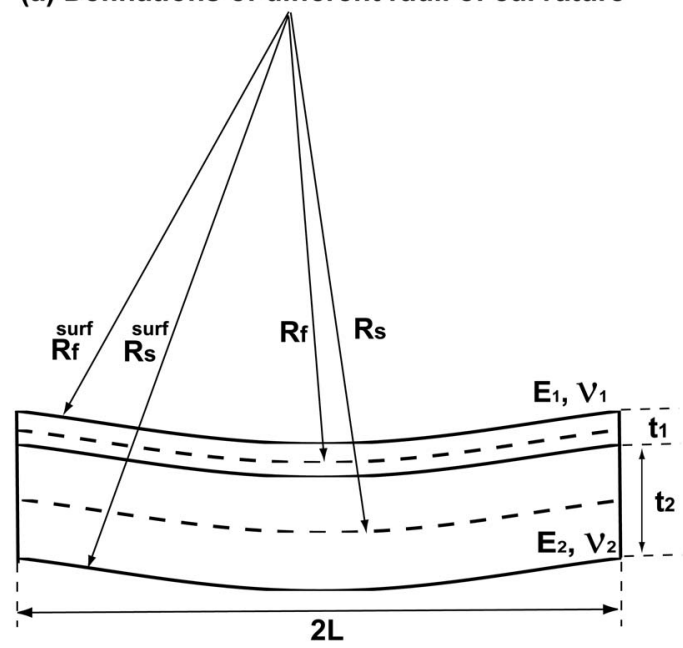

(b) Coordinate system for viewing the strain along thickness

\begin{tabular}{|l|l|}
\hline Film: $E_{1}, V_{1}$ & \multicolumn{1}{l}{} \\
\hline Substrate: $E_{2}, V_{2}$ & \\
\hline
\end{tabular}

Fig. 2 (a) The definitions of different radii of curvature. $\boldsymbol{R}_{f}$ and $\boldsymbol{R}_{s}$ are the radii of curvature of the mid-planes of film and substrate, respectively. $\boldsymbol{R}_{f}^{\text {surf }}$ and $\boldsymbol{R}_{s}^{\text {surf }}$ are the radii of curvature of the surfaces of film and substrate, respectively. (b) The coordinate system for viewing the strain along the thickness.

assumed isotropic, so $G_{o}=E_{o} / 2\left(1+\nu_{o}\right)$ [24]. $\nu_{o}$ is the Poisson's ratio of the interface layer and $\nu_{o}=0.2$ is assumed. The range of $G_{o} / \eta$ taken in Murray and Noyan's paper is around
$10^{17}-10^{21} \mathrm{~Pa} / \mathrm{m}$ [24]. Here the relatively compliant interface layer parameters are taken in order to better demonstrate the effect of interfacial slip. Two cases of $E_{o} / \eta=2 \times 10^{16} \mathrm{~Pa} / \mathrm{m}$ and 2 $\times 10^{17} \mathrm{~Pa} / \mathrm{m}$ are studied comparatively. As noticed by Huang and Zhang [15], the experimentally measured radii of curvature are those of the surfaces, which are indicated by $R_{f}^{\text {surf }}$ and $R_{s}^{\text {surf }}$ in Fig. 2(a). The beam model of both the Timoshenko and S-L model actually describes the behavior of the midplanes of the two layers. The radii of curvature given by the two models are thus $R_{f}$ and $R_{s}$ as shown in Fig. 2(a). $R_{f}=R_{f}^{\text {surf }}+t_{1} / 2$ and $R_{s}=R_{s}^{\text {surf }}-t_{2} / 2$. As demonstrated later in this section, $R_{f}$ and $R_{s}$ have the order of $10^{2}-10^{3} \mu \mathrm{m}$, the thickness $\left(t_{1}\right.$ and $\left.t_{2}\right)$ is just $1-3 \mu \mathrm{m}$. So the curvature difference between the model and experimental measurement is so little to be ignored. As mentioned above, two sets of coordinate system are used in both the Timoshenko and S-L models during the derivation. It may cause confusion when examining the strain across the thickness as shown later in Fig. 5. So here a new coordinate system located at the interface as shown in Fig. $2(b)$ is introduced for the result presentation purpose.

The curvatures of the film and substrate of the S-L model when $E_{o} / \eta=2 \times 10^{16} \mathrm{~Pa} / \mathrm{m}$ and $2 \times 10^{17} \mathrm{~Pa} / \mathrm{m}$ are calculated from Eqs. (45) and (46). The curvatures of the Timoshenko model for the film and substrate are the same. As indicated in Eq. (13), the curvature of the Timoshenko model is a constant when the dimensions are fixed. In Fig. 3, the following dimensions are used: $t_{1}$ $=t_{2}=2 \mu \mathrm{m}$ and $L=10 \mu \mathrm{m}$. The uniform curvature of the Timoshenko model is calculated as $\kappa_{T}=14914.1 \mathrm{~m}^{-1}$. For the ideal interface case described by the Timoshenko model, the curvature can only be uniform without the presence of residual axial stress [16]. During the derivation above, the implicit assumption of no residual stress is applied. For the composite with ideal interface and no residual axial stress, its curvature is proved to be independent on the length [16]. As indicated in Eq. (13), $\kappa_{T}$ of the Timoshenko model is independent of the beam length $2 L$. However, the two curvatures of the S-L model in Eqs. (45) and (46) explicitly

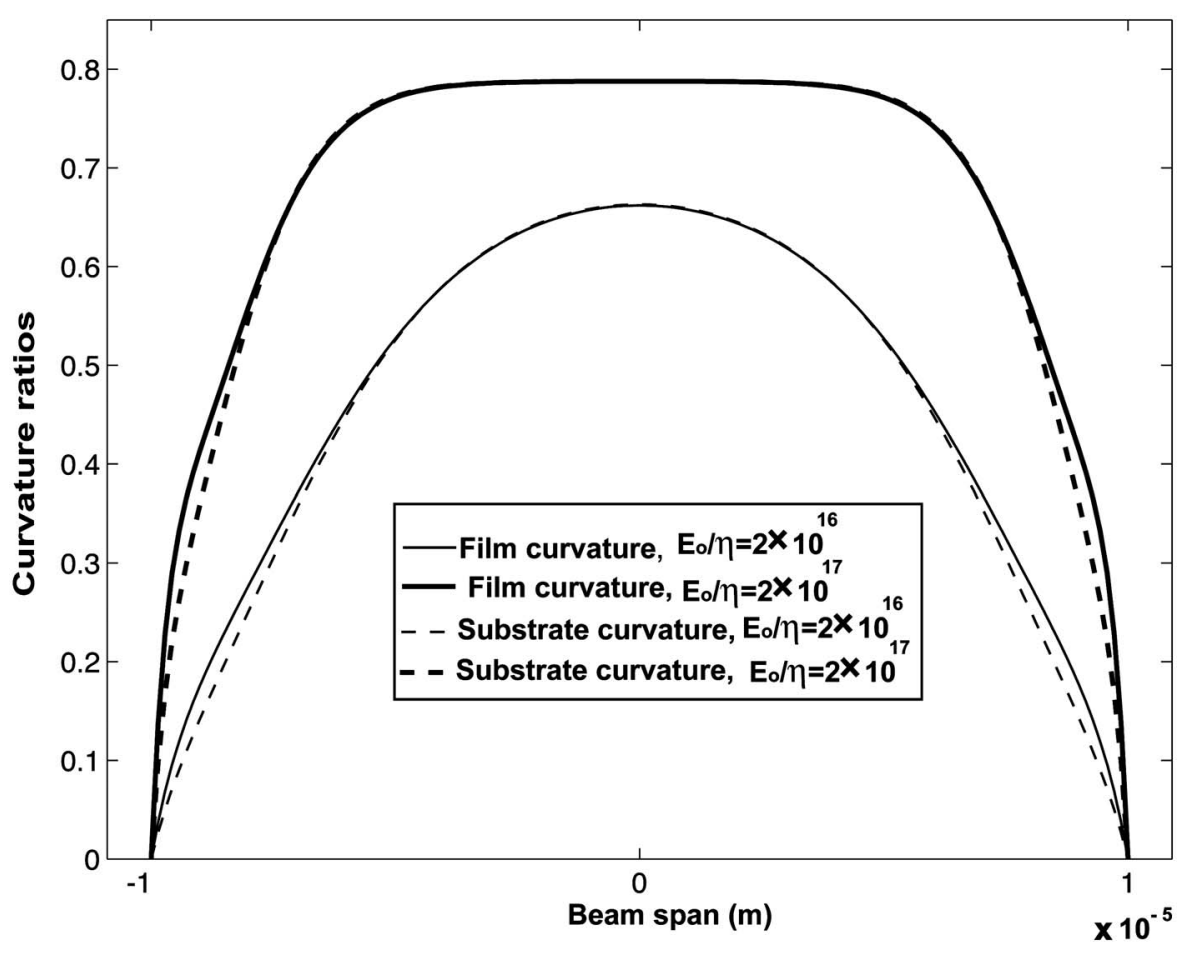

Fig. 3 The curvatures of film and substrate of the S-L model divided by $\kappa_{T}$. $E_{o} / \eta$ is taken as $2 \times 10^{16} \mathrm{~Pa} / \mathrm{m}$ and $2 \times 10^{17} \mathrm{~Pa} / \mathrm{m}$, respectively. The thickness of film layer is $t_{1}=2 \mu \mathrm{m}$ and the thickness of the substrate layer is $t_{2}=2 \mu \mathrm{m} ; L=10 \mu \mathrm{m}$ and $\kappa_{T}=14914.1 \mathrm{~m}^{-1}$. 


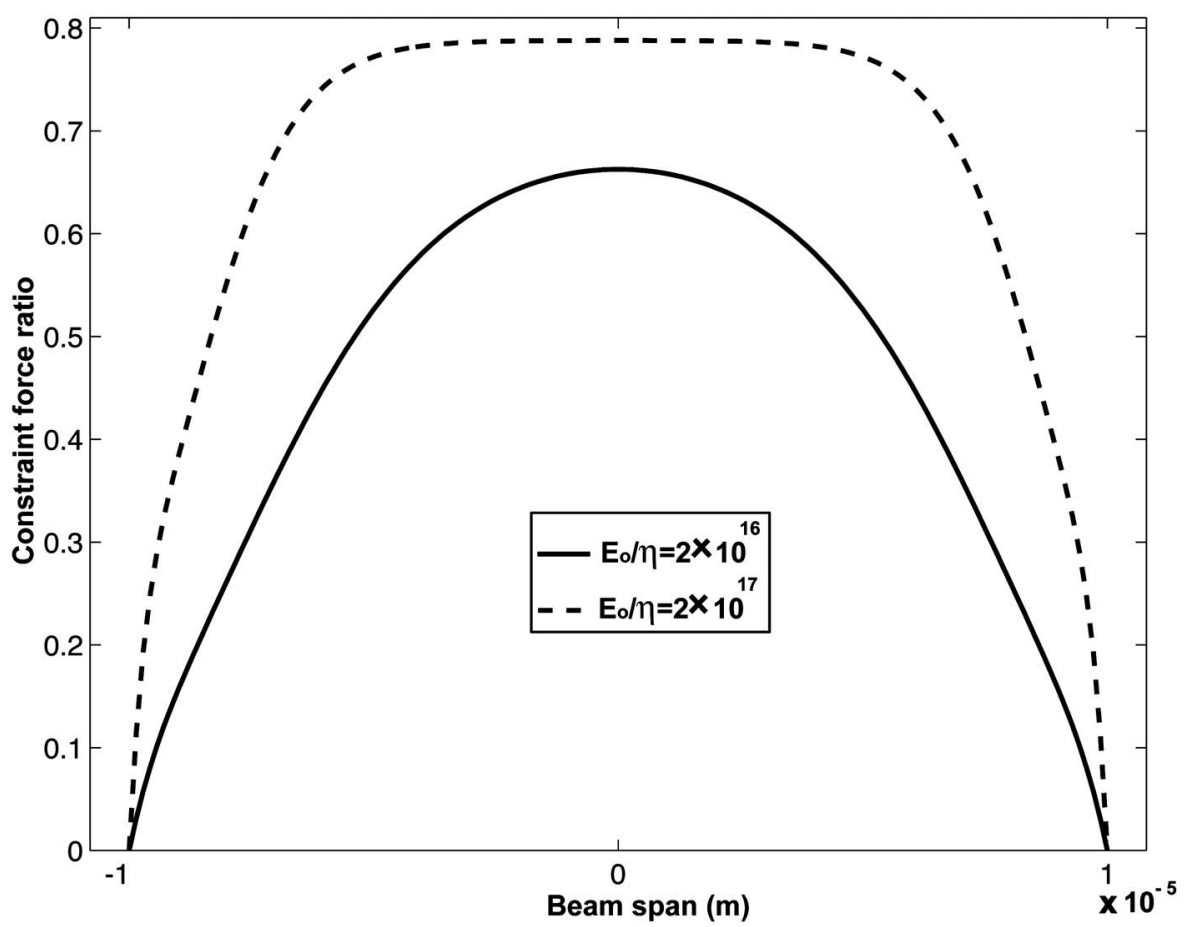

Fig. 4 The constraint forces per unit width of the S-L model with different $E_{o} / \eta$ s divided by that of the Timoshenko model $\left(P_{\text {timo }}=1268.1 \mathrm{~N} \mathrm{~m}^{-1}\right) . t_{1}=2 \mu \mathrm{m}, t_{2}=2 \mu \mathrm{m}$, and $L$ $=10 \mu \mathrm{m}$.

depend on the length. During the derivation of the Timoshenko model, no boundary conditions are used. For the composite with ideal interface and no residual axial stress, its curvature is also proved to be independent on the boundary conditions [16]. The free-free boundary conditions in Eq. (37) are used for the solution of the interfacial stresses, therefore, the curvature of the S-L model is dependent on the boundary conditions. In Fig. $3, \kappa_{f}$ and $\kappa_{s}$ are divided by $\kappa_{T}$ for comparison reason. As shown in Fig. 3, there is little difference between $\kappa_{f}$ and $\kappa_{s}$ for both $E_{o} / \eta=2$ $\times 10^{16} \mathrm{~Pa} / \mathrm{m}$ and $2 \times 10^{17} \mathrm{~Pa} / \mathrm{m}$. The $\kappa_{f}$ and $\kappa_{s}$ of $E_{o} / \eta=2$ $\times 10^{17} \mathrm{~Pa} / \mathrm{m}$ are larger than those of $2 \times 10^{16} \mathrm{~Pa} / \mathrm{m}$, respectively.

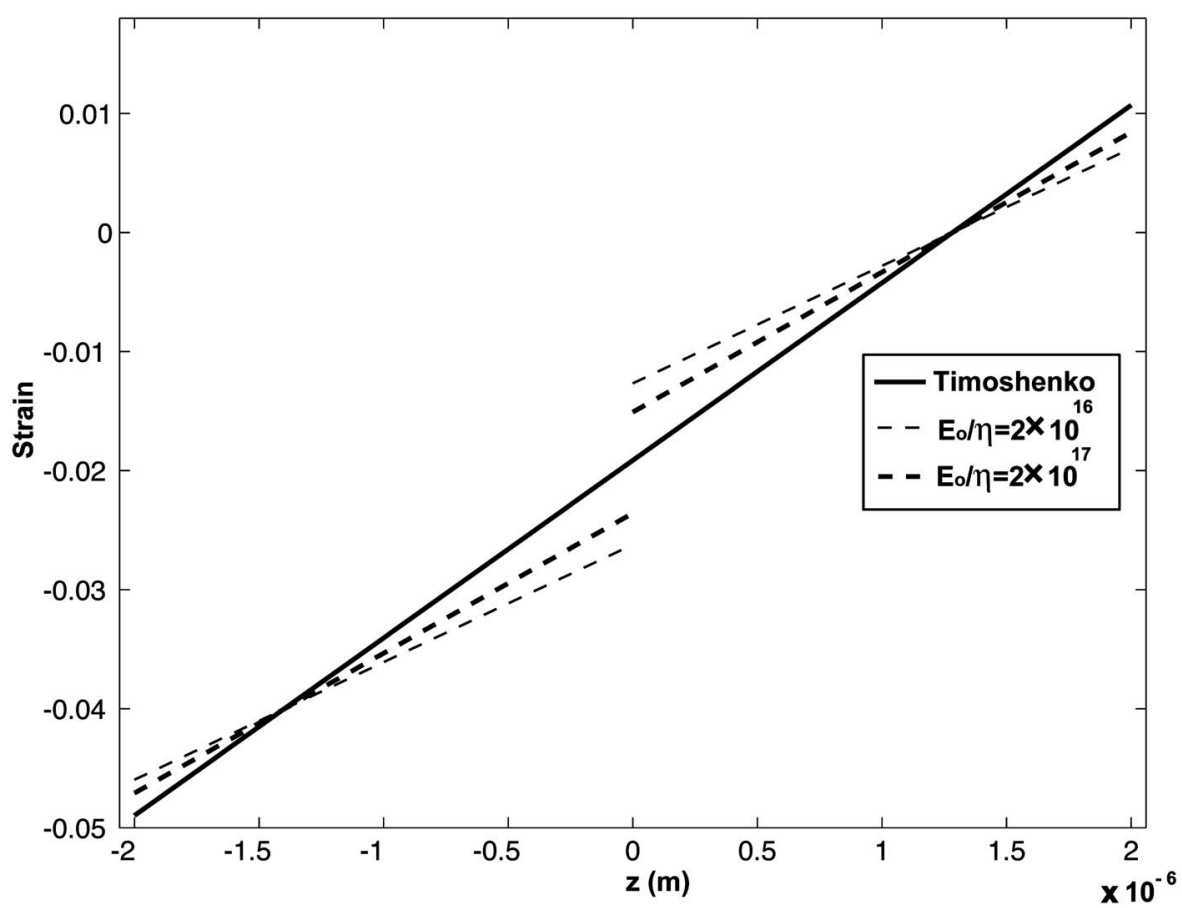

Fig. 5 The comparison of strains calculated by the Timoshenko model and S-L model with different $E_{o} / \eta \mathrm{s} . t_{1}=2 \mu \mathrm{m}, t_{2}=2 \mu \mathrm{m}$, and $L=10 \mu \mathrm{m}$. The coordinate system is given in Fig. 2(b). 


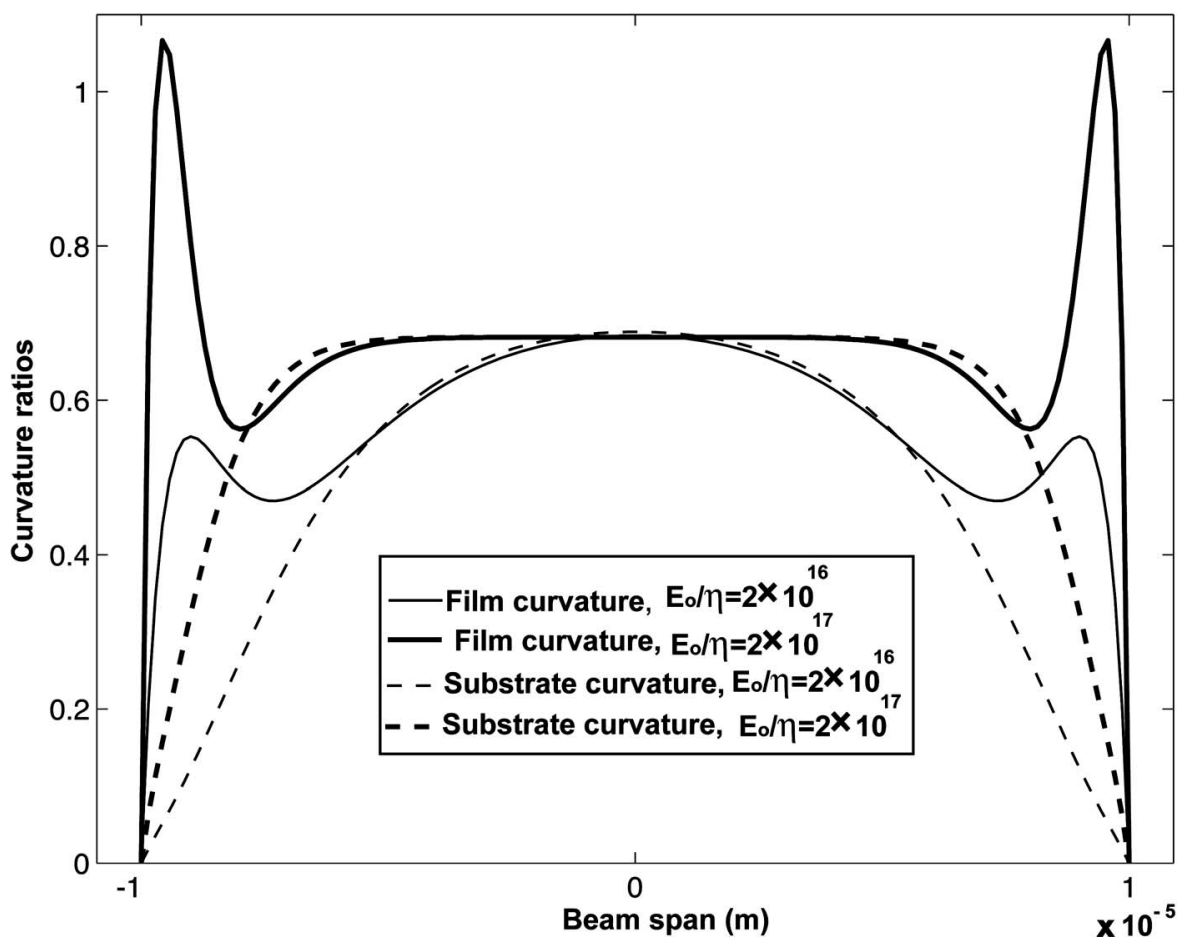

Fig. 6 The curvatures of film and substrate of the S-L model divided by $\kappa_{T}$. $E_{o} / \eta$ is taken as $2 \times 10^{16} \mathrm{~Pa} / \mathrm{m}$ and $2 \times 10^{17} \mathrm{~Pa} / \mathrm{m}$, respectively. $t_{1}=1 \mu \mathrm{m}, t_{2}=3 \mu \mathrm{m}, L=10 \mu \mathrm{m}$, and $\kappa_{T}$ $=9942 \mathrm{~m}^{-1}$.

The interface layer is less compliant with larger $E_{o} / \eta$. When $E_{o} / \eta$ approaches infinity, it is the rigid interface of the Timoshenko model, which allows no interfacial slip. It is also noticed in Fig. 3 that the curvatures of the S-L model are nonuniform. Figure 4 examines how the interfacial slip can alter the constraint axial force. The dimensions in Fig. 4 are the same as those in Fig. 3. In the Timoshenko model, the constraint axial force per unit width in Eq. (12) is solved from the constraint condition of no interfacial slip as indicated in Eq. (11). The constraint force per unit width calculated from Eq. (12) is $P_{\text {timo }}=1268.1 \mathrm{~N} \mathrm{~m}^{-1}$. The constraint

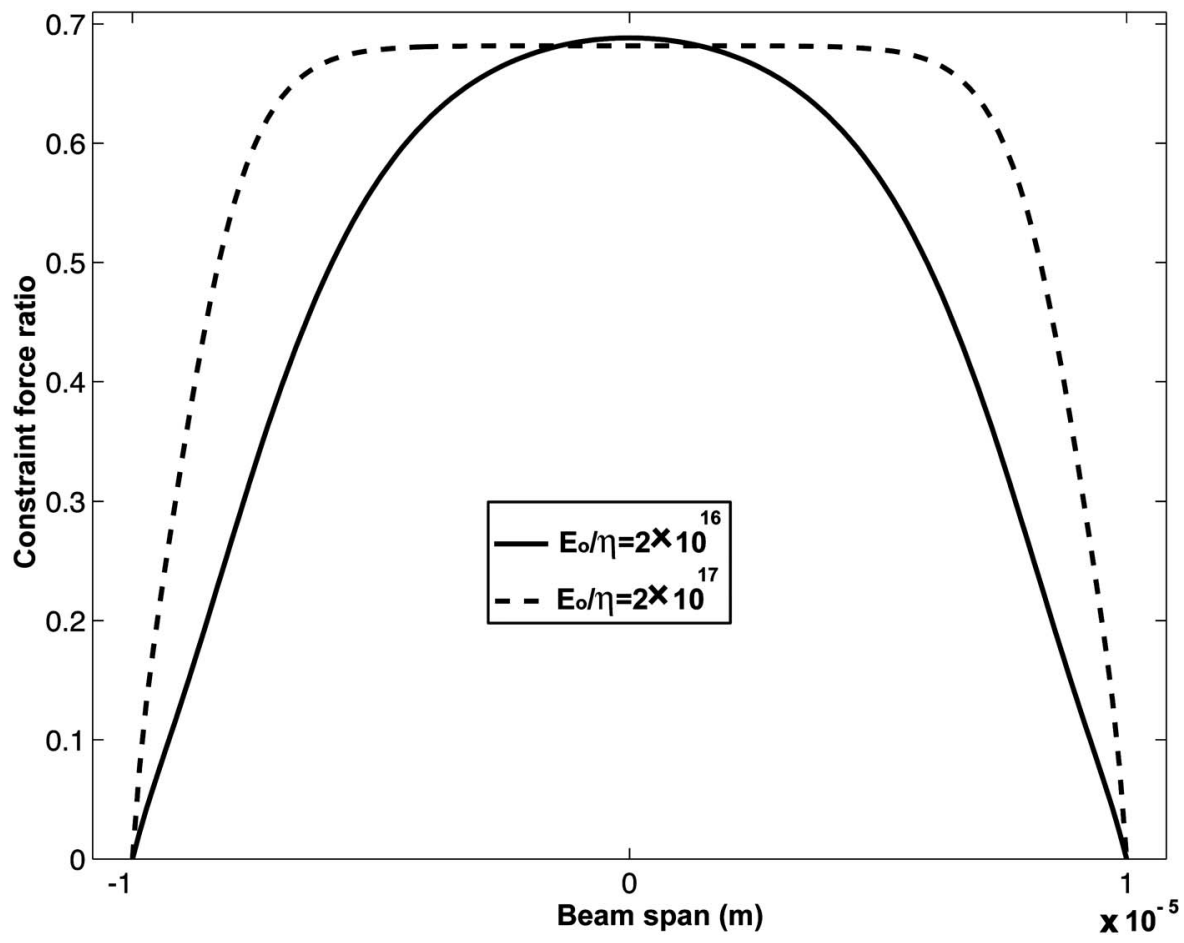

Fig. 7 The constraint forces per unit width of the S-L model with different $E_{o} / \eta$ s divided by that of the Timoshenko model $\left(P_{\text {timo }}=1721 \mathrm{~N} \mathrm{~m}^{-1}\right) . t_{1}=1 \mu \mathrm{m}, t_{2}=3 \mu \mathrm{m}$, and $L=10 \mu \mathrm{m}$. 
axial force per unit width of the S-L model is $P(x)=\int_{-L}^{x} \tau_{o} d x$ [24]. In Fig. $4, P(x)$ calculated for both $E_{o} / \eta=2 \times 10^{16} \mathrm{~Pa} / \mathrm{m}$ and 2 $\times 10^{17} \mathrm{~Pa} / \mathrm{m}$ is again divided by $P_{\text {timo }}$ for comparison. Clearly, with the interfacial slip, the constraint force is smaller than that of the ideal interface case. It is also noticed that the constraint force of the S-L model varies with $x$ and $E_{o} / \eta\left(G_{o} / \eta\right)$ with the fixed layers dimensions, while that of the Timoshenko model is a constant. Figure 5 shows the axial strains of the Timoshenko model and S-L model. The axial strain of the Timoshenko model is calculated from Eq. (8) and the S-L one is from Eq. (23). In Fig. 5, the strain of the Timoshenko model is continuous across the interface, which is also indicated by Eq. (11). The coordinate system in Fig. 5 as mentioned at the beginning of this section is the one shown in Fig. 2(b). The strains of the S-L models are discontinuous at the interface reflecting the fact of interfacial slip. The strain of $E_{o} / \eta=2 \times 10^{17} \mathrm{~Pa} / \mathrm{m}$ is less "discontinuous" than that of $E_{o} / \eta=2 \times 10^{16} \mathrm{~Pa} / \mathrm{m}$. $E_{o} / \eta$ as a fitting parameter physically indicates the effect of interfacial slip as reflected in Eq. (14). Larger $E_{o} / \eta$ means smaller interfacial slip and for ideal interface $E_{o} / \eta$ $=\infty$. As both the theoretical analysis and experiments show that the layers dimensions also have significant influence on the interfacial stresses $\left(\sigma_{o}\right.$ and $\left.\tau_{o}\right)$ as those interfacial parameters (i.e., $E_{o}$, $G_{o}$, and $\left.\eta\right)[23,24]$, so the thickness of both the film and substrate is changed in Figs. 6 and 7 to show the effect of layer dimensions. In Figs. 6 and $7, t_{1}=1 \mu \mathrm{m}, t_{2}=3 \mu \mathrm{m}$, and $L=10 \mu \mathrm{m}$. For these dimensions, $\kappa_{T}=9942 \mathrm{~m}^{-1}$ and $P_{\text {timo }}=1721 \mathrm{~N} \mathrm{~m}^{-1}$. The curvatures and constraint forces are also divided by these new $\kappa_{T}$ and $P_{\text {timo }}$, respectively. In Fig. 6, the curvature difference between the film and substrate becomes significant around the free edges. The film curvatures of both $E_{o} / \eta=2 \times 10^{16} \mathrm{~Pa} / \mathrm{m}$ and $2 \times 10^{17} \mathrm{~Pa} / \mathrm{m}$ in Figs. 6 experience much more dramatic variation around the free edges. The difference between $\kappa_{f}$ and $\kappa_{s}$ in both Figs. 3 and 6 is very little around the center. Compared with those in Fig. 4, the profiles of the film and substrate constraint forces in Fig. 7 do not have a dramatic change, just the gap (difference) around the bilayer center is smaller.

\section{Concluding Remarks}

The interfacial slip of non-ideal interface can significantly reduce the constraint force as compared with that of ideal interface. The curvature of the nonideal interface also varies with the structure length and differs from that of the ideal interface. Therefore, the evaluation of stress state inside the structure based on the measured curvature and the model of ideal interface can result in a serious error without properly evaluating the interface state. $E_{o} / \eta$ and $G_{o} / \eta$ are the fitting parameters in the S-L model to be varied to fit the experimental data. Once, the proper $E_{o} / \eta$ and $G_{o} / \eta$ are chosen, Eqs. (45) and (46) extend the Stoney's formula to the application of the composite with interfacial slip.

\section{Acknowledgment}

This work is supported by both the National Natural Science Foundation of China (NSFC, Grant No. 10502050) and the Scientific Research Foundation for the Returned Overseas Chinese Scholars, State Education Ministry of China.

\section{References}

[1] Hu, S. M., 1979, “Film-Edge-Induced Stress in Substrates,” J. Appl. Phys., 50, pp. 4661-4666.

[2] Hu, S. M., 1991, "Stress-Related Problems in Silicon Technology," J. Appl. Phys., 70, pp. R53-R80.

[3] Chen, W. T., and Nelson, C. W., 1979, "Thermal Stress in Bonded Joints," IBM J. Res. Dev., 23, pp. 179-188.

[4] Stoney, G. G., 1909, "The Tension of Metallic Films Deposited by Electrolysis," Proc. R. Soc. London, Ser. A, 82, pp. 172-175.

[5] Zhang, Y., Ren, Q., and Zhao, Y., 2004, "Modelling Analysis of Surface Stress on a Rectangular Cantilever Beam," J. Phys. D, 37, pp. 2140-2145.

[6] Freund, L. B., Floro, J. A., and Chason, E., 1999, "Extensions of the Stoney Formula for Substrate Curvature to Configurations With Thin Substrates or
Large Deformation,” Appl. Phys. Lett., 74, pp. 1987-1989.

[7] Freund, L. B., and Suresh, S., 2003, Thin Film Materials: Stress, Defect Formation and Surface Evolution, Cambridge University Press, Cambridge, UK, Chaps. 2 and 6.

[8] Timoshenko, S., 1925, "Analysis of Bi-Metal Thermostats," J. Opt. Soc. Am., 11, pp. 233-255.

[9] Brenner, A., and Senderoff, A., 1949, "Calculation of Stress in Electrodeposits From the Curvature of a Plate Strip," J. Res. Natl. Bur. Stand., 42, pp. 105123.

[10] Atkinson, A., 1995, "Macro-and Microstress Analysis in Sol-Gel Derived $\mathrm{Pb}\left(\mathrm{Zr}_{x} \mathrm{Ti}_{1-x}\right) \mathrm{O}_{3}$ Thin Films," Br. Ceram. Proc., 54, pp. 1-7.

[11] Röll, K., 1976, "Analysis of Stress and Strain Distribution in Thin Films and Substrates," J. Appl. Phys., 47, pp. 3224-3229.

[12] Hsueh, C. H., Lee, S., and Chuang, T. J., 2003, "An Alternative Method of Solving Multilayer Bending Problems,” J. Appl. Mech., 70, pp. 151-154.

[13] Blech, I. A., Blech, I., and Finot, M., 2005, "Determination of Thin-Film Stresses on Round Substrate," J. Appl. Phys., 97, p. 113525.

[14] Huang, Y., and Rosakis, A. J., 2005, "Extension of Stoney's Formula to NonUniform Temperature Distributions in Thin Film/Substrate Systems. The Case of Radial Symmetry," J. Mech. Phys. Solids, 53, pp. 2483-2500.

[15] Huang, S., and Zhang, X., 2006, "Extension of the Stoney Formula for FilmSubstrate Systems With Gradient Stress for MEMS Applications," J. Micromech. Microeng., 16, pp. 382-389.

[16] Zhang, Y., and Zhao, Y., 2006, "Applicability Range of Stoney's Formula and Modified Formulas for a Film/Substrate Bilayer," J. Appl. Phys., 99, pp. 053513 .

[17] Yang, F., and Li, J. C. M., 2003, "Diffusion-Induced Beam Bending in Hydrogen Sensors,” J. Appl. Phys., 93, pp. 9304-9309.

[18] Hu, Y. Y., and Huang, W. M., 2004, "Elastic and Elastic-Plastic Analysis of Multilayer Thin Films: Close-Form Solution,” J. Appl. Phys., 96, pp. 41544160.

[19] Wang, S. S., and Choi, I., 1982, "Boundary-Layer Effects in Composite Laminates," ASME J. Appl. Mech., 49, pp. 541-548.

[20] Cammarata, R. C., Sieradzki, K., and Spaepen, F., 2000, "Simple Model for Interface Stresses With Application to Misfit Dislocation Generation in Epitaxial Thin Films," J. Appl. Phys., 87, pp. 1227-1234.

[21] Suhir, E., 1986, "Stresses in Bi-Metal Thermostats," ASME J. Appl. Mech., 53, pp. 657-660.

[22] Suhir, E., 1989, "Interfacial Stresses in Bi-Metal Thermostats," ASME J. Appl Mech., 56, pp. 595-600.

[23] Noyan, I. C., Murray, C. E., Chey, J. S., and Goldsmith, C. C., 2004, "Finite Size Effects in Stress Analysis of Interconnect Structures," Appl. Phys. Lett., 85, pp. 724-726.

[24] Murray, C. E., and Noyan, I. C., 2002, "Finite Size Effects in Thin-Film Composites," Philos. Mag. A, 82, pp. 3087-3117.

[25] Sagalowicz, L., Rudra, A., Kapon, E., Hammar, M., Salomonsson, F., Black, A., Jouneau, P. H., and Wipijewski, T., 2000, "Defects, Structure, and Chemistry of InP-GaAs Interfaces Obtained by Wafer Bonding," J. Appl. Phys., 87, pp. $4135-4146$

[26] Liau, Z. L., 1997, "Strained Interface of Lattice-Mismatch Wafer Fusion," Phys. Rev. B, 55, pp. 12899-12901.

[27] Zhang, X., Misra, A., Wang, H., Lima, A. L., Hundley, M. F., and Hoagland, R. G., 2005, "Effects of Deposition Parameters on Residual Stresses, Hardness and Electrical Resistivity of Nanoscale Twinned 330 Stainless Steel Thin Films,” J. Appl. Phys., 97, p. 094302.

[28] Jasinski, J., Liliental-Weber, Z., Estrada, S., and Hu, E., 2002, "Microstructure of GaAs/GaN Interfaces Produced by Direct Wafer Fusion," Appl. Phys. Lett., 81, pp. 3152-3154.

[29] Shi, F., MacLaren, S., Xu, C., Cheng, K. Y., and Hsieh, K. C., 2003, "HybridIntegrated GaAs/GaAs and InP/GaAs Semiconductors Through Wafer Bonding Technology: Interface Adhesion and Mechanical Strength,” J. Appl. Phys. 93, pp. 5750-5756.

[30] Shi, F., Chang, K., Hsieh, K. C., Guido, L., and Hoke, B., 2004, "Interface Structure and Adhesion of Wafer-Bonded GaN/GaN and GaN/AlGaN Semiconductors," J. Appl. Phys., 95, pp. 909-912.

[31] Okuno, Y., Uomi, K., Aoki, M., Taniwatari, T., Suzuki, M., and Kondow, M., 1995, "Anti-Phase Direct Bonding and its Application to the Fabrication of InP-Based $1.55 \mu \mathrm{m}$ Wavelength Lasers on GaAs Substrates," Appl. Phys. Lett., 66, pp. 451-453.

[32] Ru, C. Q., 2002, "Interfacial Thermal Stresses in Bimaterial Elastic Beams: Modified Beam Models Revisited," J. Electron. Packag., 124, pp. 141-146.

[33] Benveniste, Y., 1984, "On the Effect of Debonding on the Overall Behavior of Composite Materials," Mech. Mater., 3, pp. 349-358.

[34] Tullini, N., Savoia, M., and Horgan, C. O., 1997, "End Effects in the Multilayered Orthotropic Strips With Imperfect Bonding," Mech. Mater., 26, pp. 23-34.

[35] Müller, P., and Saúl, A., 2004, "Elastic Effects on Surface Physics," Surf. Sci. Rep., 54, pp. 157-258.

[36] Timoshenko, S. P., and Woinowsky-Krieger, S. P., 1959, Theory of Plates and Shells, 2nd ed., McGraw-Hill, New York.

[37] Huang, R., and Suo, Z., 2002, "Wrinkling of a Compressed Elastic Film on a Viscous Layer," J. Appl. Phys., 91, pp. 1135-1142.

[38] Huang, R., and Suo, Z., 2002, "Instability of a Compressed Elastic Film on a Viscous Layer," Int. J. Solids Struct., 39, pp. 1791-1802. 